Pacific Journal of Mathematics

LIMIT SETS OF POWER SERIES OUTSIDE THE CIRCLES OF 


\title{
LIMIT SETS OF POWER SERIES OUTSIDE THE CIRCLES OF CONVERGENCE
}

\author{
Charles K. Chui and Milton N. Parnes
}

Let $U$ denote the open unit disc in the complex plane $C$. A power series of a complex variable with center at the origin and radius of convergence equal to one will be called a power series in $U$. It is well-known that the behaviors of a power series outside its circle of convergence are quite irregular. In particular, it is proved that for each complex number $z^{*},\left|z^{*}\right|>1$, and for every closed set $E$ in $C$, there is a power series in $U$ whose limit set at $z^{*}$ is $E$. The power series $P_{\alpha}(z)=\sum\left(1-e^{i n \alpha}\right) z^{n}, \alpha$ real, are studied in this paper. Their peculiar properties seem to suggest that it might be fruitful to study the irregularities of power series outside their circles of convergence by probability theory. To study $P_{\alpha}(z)$, results in diophantine approximation are obtained.

We first recall the following result obtained in [2]:

Theorem A. There exists a power series $P$ in $U$ with the property that for each compact set $K$ which lies outside the unit circle and has connected complement and for any function $f$ continuous on $K$ and holomorphic at the interior points of $K$, there exists a subsequence of the sequence of partial sums of $P$ that converges uniformly to $f$ on $K$.

Hence, different subsequences of the sequence of partial sums of a power series in $U$ may overconverge to different values at the same point outside the unit circle. This leads to the following

Definitions. Let $P$ be a power series in $U$ and let $|a|>1$.

(1) We denote by $L(a, P)$ the set of all complex values which are the limits of all the convergent subsequences of the sequence of partial sums of $P$ at the point $a$ and we call it the limit set of $P$ at $a$.

(2) If $L(a, P) \neq \varnothing$ where $|a|>1$, we say that $a$ is an exceptional point of the power series $P$.

For instance, if $P(z)=\sum_{k=0}^{\infty} z^{k}$ is the geometric series, it is clear that $L(\alpha, P)=\varnothing$ for every point $a$ with $|a|>1$, so that the geometric series has no exceptional points outside the unit circle. On the other hand, if $P$ is a "universal series" as described in Theorem A, then every point $a$ outside the unit circle is an exceptional point of $P$ and $L(a, P)=C$, the entire complex plane. We first establish the following result. 
THEOREM 1. For every complex number a of absolute value greater than one and for every closed set $E$ in the complex plane, there is $a$ power series $P$ in $U$ such that $L(a, P)=E$.

Next, we observe that the behavior of a power series outside its circle of convergence is like a "random walk". If $P$ is a power series in $U$ with coefficients $a_{n}$ and if $|z|>1$, then $\lim \sup \left|a_{n} z^{n}\right|=\infty$ so that we can interpret that the walk of $P(z)$ has "very large steps". If $P$ has no exceptional point, the walk of $P(z),|z|>1$, can only lead to infinity. On the other hand, $z_{0}$ is an exceptional point of $P$ if and only if the walk of $P\left(z_{0}\right)$ enters a finite neighborhood infinitely often.

We prove two more theorems in $\S 2$. Theorem 2 gives a criteria in terms of the coefficients of the power series to determine a region of the plane with no exceptional points, and Theorem 3 contains an analysis of when certain gap series have no exceptional points. The entire section is very elementary and uncomplicated. In $\S 3$ we prove some results on diophantine equations, most of which are needed to prove Theorem 6 of $\S 4$.

The theorems we would like to have proved would say that if a series is "active" enough to have exceptional points then $L(z, P)$ will usually be "as large as possible". We have no theorems of this sort but only an example. The example is stated as Theorem 6 in $\S 4$. Let

$$
f_{\alpha}(z)=\frac{1}{1-z}-\frac{1}{1-z e^{i \alpha}}
$$

where $0<\alpha<2 \pi$ and let $P_{\alpha}$ denote the power series expansion of $f_{\alpha}$ in $U$. Then for all irrational multiple, $\alpha$, of $2 \pi$ the power series $P_{\alpha}$ has the property that the set of exceptional points $z=\mathrm{Re}^{-i \alpha / 2}$ with corresponding limit sets

$$
L\left(z, P_{\alpha}\right)=\left\{f_{\alpha}(z)+\frac{i e^{-i \lambda / 2}}{1-z} x: \quad x \text { real }\right\}
$$

where

$$
\lambda=\arg \left\{\left[1-\operatorname{Re}^{i \alpha / 2}\right] /\left[1-\operatorname{Re}^{-i \alpha / 2}\right]\right\}
$$

and $0 \leqq \lambda<2 \pi$, is uncountable and dense in the ray $\mathrm{Re}^{-i \alpha / 2}, R>1$. It seems to be a promising evidence for the type of result we seek. However, in Theorem $6^{*}$ of $\S 5$ we present a version of Theorem 6 which seems to run counter to this view. In $\S 6$ we shall try to justify why we believe that Theorem $6^{*}$ does not necessarily run counter to the sort of theorem we should like to have proved.

We close our introduction with a historical note. Around the 
year 1910, R. Jentzsch started the study of the zeros of the sections $P_{n}$ of a power series $P$ in $U$; and the related development of the phenomenon of overconvergence was discovered by M. B. Porter in 1906 and was later rediscovered by R. Jentzsch in 1914 and A. Ostrowski in 1921 [cf. 4 and 5]. Since then, this work has been much generalized and followed up by many mathematicians. For instance, there are fifteen articles in the Japanese Journal of Mathematics between 1924 and 1928, contributed by S. Izumi, S. Narumi, T. Shimizu, and M. Tsuji. Their results are somewhat related (although from a different point of view) to ours in this paper.

2. Proof of Theorem 1; coefficient and gap series theorems. To prove Theorem 1, we let $|a|>1$ and let $E$ be any closed set in the complex plane. Let $\left\{b_{j}\right\}$ be a dense sequence of points in $E$. We construct the power series $P(z)=\sum_{k=0}^{\infty} a_{k} z^{k}$ in $U$ as follows:

Let $P_{n}(z)=\sum_{k=0}^{n} a_{k} z^{k}$ denote the $(n+1)$ th partial sum of $P$. We choose a sequence of integers $\left\{k_{n}\right\}, 1 \leqq k_{1}<k_{2}<\cdots$, inductively so that $|a|^{k_{1}} \geqq\left|b_{1}\right|$, and for $n=2,3, \cdots$,

$$
|a|^{k_{n}} \geqq \sum_{j=1}^{n-1}\left|P_{k_{j}}(a)\right|+\sum_{j=1}^{n}\left|b_{j}\right|,
$$

where we choose simultaneously $a_{j}=0$ if $j$ is not one of the $k_{n}$ $(n=1,2, \cdots)$, and for each $j=1,2, \cdots$, we take $a_{k_{2 j-1}}=1$ and define, inductively, $a_{k_{2 j}}=\left(1 / a^{k_{2 j} j}\right)\left(b_{j}-P_{k_{2 j}-1}(a)\right)$. Hence, $P_{k_{2 j}}(a)=b_{j}$ and $P_{k_{2 j-1}}(a)=P_{k_{2 j-1}}(a)=a^{k_{2 j-1}}+P_{k_{2(j-1)}}(a)$, where $j=1,2, \cdots$. Therefore, for each $j=1,2, \cdots$,

$$
\begin{aligned}
\left|a_{k_{2 j}}\right| & \leqq\left(1 /|a|^{k_{2 j}}\right)\left(\left|b_{j}\right|+\left|P_{2 j-1}(a)\right|\right) \\
& \leqq\left(1 /|a|^{k_{2 j}}\right)\left(\sum_{r=1}^{2 j}\left|b_{r}\right|+\sum_{r=1}^{2 j-1}\left|P_{k_{r}}(a)\right|\right) \\
& \leqq\left(1 /|a|^{k_{2 j} j}\right)|a|^{k_{2 j}}=1 .
\end{aligned}
$$

Also, the other coefficients $a_{r}$ of the power series $P$ are either zero or one and the coefficient one appears infinitely often. Thus, $P$ is a power series in $U$.

Now for each $b \in E$, let $\left\{b_{j(n)}\right\}$ be a subsequence of our dense sequence $\left\{b_{j}\right\}$ which converges to $b$. Since $P_{k_{2 j(n)}}(a)=b_{j(n)}$, we see that $b \in L(a, P)$. Hence, $E \subset L(a, P)$. On the other hand, for each integer $N, P_{N}(a)=P_{k_{n}}(a)$ where $k_{n} \leqq N<k_{n+1}$. If $n$ is odd, say $n=2 j+1$, then $P_{N}(a)=P_{k_{2 j+1}}(a)=a^{k_{2 j+1}}+P_{k_{2 j}}(a)=a^{k_{2 j+1}}+b_{j}$, so that

$$
\begin{aligned}
\left|P_{N}(a)\right| & \geqq|a|^{k_{2 j+1}}-\left|b_{j}\right| \geqq|a|^{k_{2 j+1}}-\sum_{r=1}^{j-1}\left|P_{k_{r}}(a)\right|-\sum_{r=1}^{j}\left|b_{r}\right| \\
& \geqq|a|^{k_{2 j+1}}-|a|^{k_{j \rightarrow \infty}} .
\end{aligned}
$$


If $n$ is even, say $n=2 j$, then $P_{N}(a)=P_{k_{2 j}}(a)=b_{j} \in E$. Since $E$ is a closed set, we have $L(a, P) \subset E$, so that $L(a, P)=E$.

We like to point out that this result is quite specialized in that we construct a different series for each point $a,|a|>1$, and for each closed set $E$.

Next, let us consider a class of all power series in $U$ with bounded coefficients:

$$
P(z)=\sum_{k=0}^{\infty} a_{k} z^{k}, \quad\left|a_{k}\right| \leqq M, \quad k=0,1, \cdots .
$$

We observe that a subsequence of the coefficients of a power series $P$ of this class may have very small absolute values, so that even though $|z|>1$, some steps of the walk $P(z)$ may be small enough that the walk may enter a finite neighborhood infinitely often. For instance the coefficients of $P_{\alpha}, \alpha / 2 \pi$ irrational, are uniformly bounded by 2 and the exceptional points of $P_{\alpha}$ are dense on the infinite ray $\operatorname{Re}^{-\alpha / 2}, R>1$, (cf. $\S 4$ ). Hence, in order that the walk of the power series

$$
P(z)=\sum_{k=0}^{\infty} a_{k} z^{k}
$$

does not enter any finite neighborhood infinitely often for large values of $|z|>1$, we also require a positive lower bound for the nonzero $\left|a_{k}\right|, k=0,1, \cdots$. We have

THEOREM 2. Let

$$
P(z)=\sum_{k=0}^{\infty} a_{k} z^{n_{k}}
$$

where $0 \leqq n_{0}<n_{1}<\cdots$ and $0<m \leqq\left|a_{k}\right| \leqq M<\infty$ for all $k=0,1, \cdots$. Then $P$ has no exceptional points in $|z| \geqq r_{0}$, where $r_{0}=1+M / m$. Furthermore, the value $r_{0}=1+M / m$ is best possible.

Proof. Let

$$
P(z)=\sum_{k=0}^{\infty} a_{k} z^{n_{k}}
$$

where $0 \leqq n_{0}<n_{1}<\cdots$ and $0<m \leqq\left|a_{k}\right| \leqq M<\infty$ for all $k=0,1, \cdots$. Let $P_{n}$ be the $(n+1)$ th partial sum of $P$ and $r_{0}=1+M / m$. Suppose that for some $z$ with $|z|=r \geqq r_{0}$, there exists a number $B<\infty$ such that $\left|P_{n}(z)\right|<B$ for a subsequence of $n$. We write this subsequence as $n=n_{k_{j}}=N_{j}, j=1,2, \cdots$. Then $\left|P_{N_{j}}(z)\right|<B, j=1,2, \cdots$. Now for each $j$, we write

$$
P_{N_{j+1}}(z)=P_{N_{j}}(z)+\sum_{t=\kappa_{j}+1}^{k_{j+1}-1} a_{t} z^{n_{t}}+a_{k_{j+1}} z^{N^{N} j+1}
$$


and hence,

$$
B>\left|P_{N_{j+1}}(z)\right|>-B+m|z|^{N_{j+1}}-M \sum_{t=k_{j}+1}^{k_{j+1}-1}|z|^{n_{t}} .
$$

Since $n_{k_{j}+1}>n_{k_{j}}=N_{j}$ and $n_{k_{j+1}-1} \leqq n_{k_{j+1}}-1=N_{j+1}-1$, we have

$$
m r^{N_{j+1}}-M \sum_{i=N_{j}}^{N} r^{+1^{-1}}<2 B .
$$

Now, $r \geqq r_{0}$ implies $-M /(r-1) \geqq-M /\left(r_{0}-1\right)=-m$. Hence, we have

$$
M r^{N} j /(r-1)<2 B,
$$

which is a contradiction if we take $j \rightarrow \infty$.

To prove that the value $r_{0}=1+M / m$ is best possible, we concider the following example. Let

$$
p_{n}(x)=m x^{n}-M\left(x^{n-1}+\cdots+1\right) .
$$

Then $p_{n}(1)=m-n M<0$ and $p_{n}\left(r_{0}\right)=m(1+M / m)^{n}-\left[m(1+M / m)^{n}-1\right]=$ $1>0$. Hence, considered as a polynomial of one real variable, $p_{n}$ has at least one zero in $\left(1, r_{0}\right)$. Let $x_{n}$ be the largest zero of $p^{n}$ in $\left(1, r_{0}\right)$. Then

$$
m x_{n}^{n}=M\left(x_{n}^{n}-1\right) /\left(x_{n}-1\right) .
$$

Hence, $x_{n}^{n}=M /\left[m\left(r_{0}-x_{n}\right)\right]$. Since $p_{n}(1) \rightarrow-\infty, x_{n}^{n} \rightarrow \infty$ or $x_{n} \rightarrow r_{0}$. Let $c$ be any real number such that $1<c<r_{0}$. Then there is an $n^{\prime}$ such that $p_{n^{\prime}}$ has a zero in $\left(c, r_{0}\right)$. Let $x_{0}$ be this zero. We now construct our power series $P$ as

$$
P(z)=\sum_{k=0}^{\infty} a_{k} z^{n_{k}}=\sum_{j=0}^{\infty} p_{n^{\prime}}(z) z^{N_{j}}
$$

where the $N_{j}$ are so chosen that $N_{j}-N_{j-1}>n^{\prime}, j=1,2, \cdots$. Hence, the coefficients $a_{k}$ can only be $m$ or $-M$ so that $0<m \leqq\left|a_{k}\right| \leqq$ $M<\infty, k=0,1, \cdots$. Furthermore, since $p_{n^{\prime}}\left(x_{0}\right)=0$, the power series $P$ overconverges to zero at the point $x_{0}$. That is, the power series $P$ has an exceptional point $x_{0} \in\left(c, r_{0}\right)$. Since $c$ is arbitrarily chosen in $\left(1, r_{0}\right)$, we have completed the proof of the theorem.

Now, let us consider the class of all gap series

$$
P(z)=\sum_{k=0}^{\infty} a_{k} z^{n_{k}}
$$

where $\left|a_{k}\right|=1, k=0,1,2, \cdots$ and $0 \leqq n_{0}<n_{1}<\cdots$. Since $\left|a_{k}\right|=1$ for all $k$, the magnitudes of the steps of the walk of $P(z)$ depend only on $|z|^{n_{k}},|z|>1$. Hence, one would expect that if the sequence 
$\left\{n_{k}\right\}$ tends to infinity very rapidly, the power series $P$ should not have any exceptional point in $|z|>1$. However, let us consider the following example.

Let $p(z)=z^{2}-z-1$. Then if $z_{0}=(1+\sqrt{5}) / 2,\left|z_{0}\right|>1$ and $p\left(z_{0}\right)=0$. Consider the gap series

$$
P(z)=\sum_{k=0}^{\infty} z^{n_{k}}=\sum_{k=0}^{\infty} p(z) z^{N_{k}}
$$

where $N_{k}-N_{k-1} \geqq 3, k=1,2, \cdots$. Hence, $0 \leqq n_{0}<n_{1}<\cdots$. It is clear that $P$ has an exceptional point at $z_{0}$ even though the sequence $\left\{N_{k}\right\}$, and hence $\left\{n_{k}\right\}$, is allowed to tend to infinity arbitrarily fast. For instance, a Fabry gap series (with $\lim _{k \rightarrow \infty} k / n_{k}=0$ ) and a gap series with $\sum_{k=1}^{\infty} 1 / n_{k}<\infty$ may have exceptional points in $|z|>1$.

However, if the sequence $\left\{n_{k}\right\}$ tends to infinity "uniformly fast", say $\left(n_{k}-n_{k-1}\right), k=1,2, \cdots$, are also large, then the walk of the gap series $P(z),|z|>1$, has to lead to infinity. We have the following

THEOREM 3. Let

$$
P(z)=\sum_{k=0}^{\infty} a_{k} z^{n k}
$$

where $\left|a_{k}\right|=1, k=0,1,2, \cdots$ and $0 \leqq n_{0}<n_{1}<\cdots$. Then the power series $P$ has no exceptional points in $|z|>1$, provided that any of the following gap conditions is satisfied:

(a) $n_{k} / n_{k-1} \geqq \lambda>1, k=1,2, \cdots$.

(b) $n_{k}-n_{k-1} \rightarrow \infty$ as $k \rightarrow \infty$.

(c) Let $\alpha>1$ be arbitrarily chosen. For each $r>1$, there is an $N_{r}$, such that for all $k \geqq N_{r}$,

$$
n_{k}-n_{k-1} \geqq \alpha-\log (r-1) / \log r .
$$

(d) For each $r>1$,

$$
r^{n_{k}}-\frac{r}{r-1} r^{n_{k-1}} \rightarrow \infty
$$

The proof of this theorem is easy. It is clear that $(a) \Rightarrow(b) \Rightarrow(c)$. We also remark that $(c) \Rightarrow(d)$. Indeed, if (c) is satisfied, then for $r>1$ and $k \geqq N_{r}$, we have

$$
r^{\left(n_{k}-n_{k-1}\right)} \geqq \frac{r^{\alpha}}{r-1}
$$

where $\alpha>1$, so that

$$
r^{n_{k-1}}\left[r^{\left(n_{k}-n_{k-1}\right)}-\frac{r}{r-1}\right] \rightarrow \infty .
$$


Hence, $(\mathrm{c}) \Rightarrow(\mathrm{d})$. Now suppose that $(\mathrm{d})$ is satisfied. Then for $|z|=$ $r>1$, we have

$$
\begin{aligned}
\left|a_{0} z^{n_{0}}+\cdots+a_{k} z^{n_{k}}\right| & >r^{n_{k}}-\left(r^{n_{0}}+\cdots+r^{n_{k-1}}\right) \\
& >r^{n_{k}}-r^{n_{k-1}}\left(1+r^{-1}+r^{-2}+\cdots\right) \\
& =r^{n_{k}}-\frac{r}{r-1} r^{n_{k-1}} \rightarrow \infty .
\end{aligned}
$$

That is, $P$ has no exceptional point in $|z|>1$.

3. Results in related diophantine approximation. The following investigation shall be used in $\S 4$. Let $R(x)>1$ be a nonincreasing function on the closed interval $[r, s] \subset(0,1)$. As usual (cf. [1]), let $\{x\}$ be the fractional part of the real number $x$, that is, $\{x\}=x-$ $[x]$, where $[x]$ is the largest integer less than or equal to $x$. Let $a \in(0,1)$ be a irrational number and let $b \in(r, s)$. We must investigate the diophantine inequality

$$
\frac{B}{R(b)^{n}} \leqq\{n a\}-b \leqq \frac{A}{R(b)^{n}}
$$

where $A$ and $B$ are arbitrarily chosen real numbers and $n=1,2, \cdots$. Let $\theta_{R}(a ; B, A)$ be the set of $b \in[r, s]$ such that there exist infinitely many $n$ satisfying (1), and let

$$
\theta_{R}(\alpha)=\bigcup_{B<A} \theta_{R}(\alpha ; B, A) .
$$

Proposition 1. For any irrational number $a$ on $(0,1)$, the set $\theta_{R}(\alpha ; B, A)$ is of cardinality $c$ (that of the continuum), of measure zero and dense in $[r, s]$.

Proof. Let $B_{n}$ be the set of values $b$ satisfying (1), and let $\mu\left(B_{n}\right)$ be the Lebesgue measure of $B_{n}$. Then $\mu\left(B_{n}\right) \leqq(A-B) / R(s)^{n}$. If $b$ is a solution to (1) for more than $K$ different $n$ 's, then $b \in$ $\bigcup_{n=K}^{\infty} B_{n}$. Hence, $\theta_{R}(a ; B, A)$ is a subset of $\bigcup_{n=K}^{\infty} B_{n}$ for any $K$, and

$$
\mu\left(\theta_{R}(\alpha ; B, A)\right) \leqq \mu\left(\bigcup_{n=K}^{\infty} B_{n}\right) \leqq \sum_{n=K}^{\infty} \frac{A-B}{R(s)^{n}}
$$

for any $K$, so that $\mu\left(\theta_{R}(a ; B, A)\right)=0$.

To show that $\theta_{R}(a ; B, A)$ is dense in $[r, s]$, we take $I_{1}=\left[a_{1}, b_{1}\right] \subset$ $(r, s)$ where $a_{1}<b_{1}$; and by the density of the sequence $\{n a\}$ in $(0,1)$ and the fact that $1 / R^{n}(s)$ tends to zero, we can find an $n_{1}$ such that $\left\{n_{1} a\right\}-A / R\left(a_{1}\right)^{n_{1}},\left\{n_{1} a\right\}-B / R\left(a_{1}\right)^{n_{1}},\left\{n_{1} a\right\}-A / R\left(b_{1}\right)^{n_{1}}$ and $\left\{n_{1} a\right\}-B / R\left(b_{1}\right)^{n_{1}}$ all lie in $I_{1}$. (For convenience, we assume that $A$ and $B$ are both positive. If they are both negative we have a similar proof, and if 
$B$ is negative and $A$ is positive the proof is even easier.) Then from (1) and the fact that $R\left(a_{1}\right) \geqq R(x) \geqq R\left(b_{1}\right)$ for any $x \in I_{1}$, we have the following inequalities

(2) $\left\{n_{1} a\right\}-A / R\left(a_{1}\right)^{n_{1}} \geqq\left\{n_{1} a\right\}-A / R(x)^{n_{1}} \geqq\left\{n_{1} a\right\}-A / R\left(b_{1}\right)^{n_{1}}$ where each respective quantity in (2) is smaller than the corresponding quantity of the inequalities

(2') $\left\{n_{1} a\right\}-B / R\left(a_{1}\right)^{n_{1}} \geqq\left\{n_{1} a\right\}-B / R(x)^{n_{1}} \geqq\left\{n_{1} a\right\}-B / R\left(b_{1}\right)^{n_{1}}$. Suppose that

(3) $\left\{n_{1} a\right\}-B / R\left(b_{1}\right)^{n_{1}}>\left\{n_{1} a\right\}-A / R\left(a_{1}\right)^{n_{1}}$ holds. Then (1) holds for all $x$ (in place of $b$ ) in $I_{1}$ and in this case, we let

(4) $\quad a_{2}=\left\{n_{1} a\right\}-A / R\left(b_{1}\right)^{n_{1}}, b_{2}=\left\{n_{1} a\right\}-B / R\left(a_{1}\right)^{n_{1}}$ and take $I_{2}=\left[a_{2}, b_{2}\right]$. Since $I_{2} \subset I_{1},(1)$ holds for $n=n_{1}$ and for all $x$ (in place of $b$ ) in $I_{2}$.

Suppose that (3) does not hold. Then we have to find $\widetilde{a}_{1}$ and $\widetilde{b}_{1}$ such that $a_{1}<\widetilde{a}_{1}<\widetilde{b}_{1}<b_{1}$ and

(5) $\left\{n_{1} a\right\}-A / R\left(\widetilde{a}_{1}\right)^{n_{1}}<\left\{n_{1} a\right\}-B / R\left(\widetilde{b}_{1}\right)^{n_{1}}$. To show that we can find such $\widetilde{a}_{1}$ and $\widetilde{b}_{1}$, we consider the following figure:

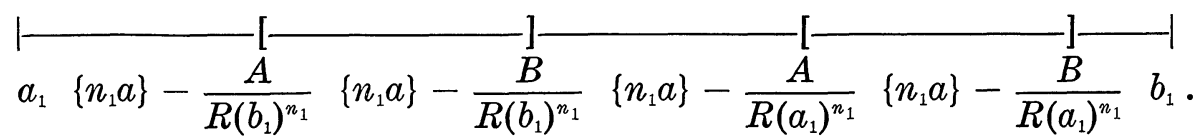

When we move $a_{1}$ slightly to the right to search for $\widetilde{a}_{1}$, the interval $\left[\left\{n_{1} a\right\}-A / R\left(a_{1}\right)^{n_{1}},\left\{n_{1} a\right\}-B / R\left(a_{1}\right)^{n_{1}}\right]$ moves to the left to become the interval $\left[\left\{n_{1} a\right\}-A / R\left(\widetilde{a}_{1}\right)^{n_{1}},\left\{n_{1} a\right\}-B / R\left(\widetilde{a}_{1}\right)^{n_{1}}\right]$. Similarly, when we move $b_{1}$ slightly to the left to search for $\widetilde{b}_{1}$, the interval $\left[\left\{n_{1} a\right\}-A / R\left(b_{1}\right)^{n_{1}},\left\{n_{1} a\right\}-B / R\left(b_{1}\right)^{n_{1}}\right]$ moves to the right to become the interval $\left[\left\{n_{1} a\right\}-A / R\left(\widetilde{b_{1}}\right)^{n_{1}},\left\{n_{1} a\right\}-B / R\left(\widetilde{b}_{1}\right)^{n_{1}}\right]$. It is not difficult to see that we can move $a_{1}$ and $b_{1}$ as above such that the new intervals overlap and $\widetilde{a}_{1}<\widetilde{b}_{1}$, where we choose either

$$
\widetilde{a}_{1}=\sup \left\{x \in I_{1}: x<\left\{n_{1} a\right\}-\frac{A}{R(x)^{n_{1}}}\right\} \text { and } \quad \tilde{b}_{1}=b_{1}
$$

or

$$
\tilde{a}_{1}=a_{1} \text { and } \quad \widetilde{b}_{1}=\inf \left\{x \in I_{1}: x>\left\{n_{1} a\right\}-\frac{B}{R(x)^{n_{1}}}\right\} .
$$

We then let

$$
\widetilde{a}_{2}=\left\{n_{1} a\right\}-A / R\left(\widetilde{b_{1}}\right)^{n_{1}}, \quad{\widetilde{b_{2}}}=\left\{n_{1} a\right\}-B / R\left(\widetilde{a}_{1}\right)^{n_{1}}
$$

and take $I_{2}=\left[\widetilde{a}_{2}, \widetilde{b}_{2}\right]$. Then $I_{2} \subset I_{1}$ and (1) holds for all $x$ (in place of $b$ ) in $I_{2}$ for $n=n_{1}$. Hence, in any case, we can define $I_{2}$ to have 
the desired properties. Now, we proceed inductively to define $I_{3}$ in a similar way, and so on. Then $I_{1} \supset I_{2} \supset \cdots$, and diam $I_{n} \rightarrow 0$. Let $b$ be the common intersection of all the $I_{n}$.

However, our interval $I_{1}$ was chosen arbitrarily. Hence, the set $\theta_{R}(a ; B, A)$ of the elements $b$ is dense in $[r, s]$.

By modifying the proof a little, we can actually prove that the cardinality of $I_{1} \cap \theta_{R}(a ; B, A)$ is $c$. This is done by using the familiar technique of constructing nested nondegenerated closed intervals such that each closed interval contains two disjoint ones. Hence, every chain of nested nonempty intervals converge to a different point and there are $c$ many different chains. This completes the proof of the proposition.

REMARK. Since all proofs concerning measures, density and cardinality are similar to the above proof, we shall only devote our attention to proving existence in the remaining propositions.

It is clear that $\theta_{R}(\alpha)=\bigcup_{n=1}^{\infty} \theta_{R}(a ;-n, n)$, so that we have the following

COROLLARY. If a is irrational, then $\theta_{R}(a)$ is of cardinality $c$, of measure zero and dense on $[r, s]$.

Next, we make the following

Definition. Let $L^{*}(b, a)$ be the set of all accumulation points of the sequence $k_{n}$ where $k_{n}$ is defined by $\{n a\}-b=k_{n} / R(b)^{n}$. If $L^{*}(b, a)$ is the whole real line, we say that $L^{*}(b, a)$ is full.

Proposition 2. For any irrational a on $(0,1)$, the set of $b$, such that $L^{*}(b, a)$ is full, is of cardinality $c$, of measure zero and dense on $[r, s]$.

Proof. Let $r_{1}, r_{2}, \cdots$ be a dense sequence of real numbers. We proceed by induction as in the proof of the above proposition, except that we change the constants $A$ and $B$ to be the constants $r_{n}+1 / n$ and $r_{n}-1 / n$ respectively in the $n$th induction step. It then follows routinely that this will work.

We refer to the final section for further comments on the above proposition.

We now close this section with a result in a slightly different direction. If in (1) we hold $b$ fixed instead, then we have the following

Proposition 3. Let $0<b<1$ and let $f$ be a positive function 
defined on the positive integers. Then there are uncountably many values of a with the property that for each of these a, there exist infinitely many pairs of integers $n$ and $m_{n}$ such that $\left|n a-b-m_{n}\right|<$ $n f(n)$. If $\sum f(n)<\infty$, the set of values $a$ is of measure zero.

Proof. Choose $a_{0}$ and $\varepsilon_{0}$ such that $0<\varepsilon_{0}<f(1)$, and that $a_{0}=$ $b-\varepsilon_{0}$ is a nonnegative rational number. Let $n_{0}=1$, and define, inductively, the nonnegative rational numbers $a_{j}$, the positive $\varepsilon_{j}$ and the positive integers $n_{j}$, such that for each $k=1,2, \cdots$,

$$
\frac{b}{n_{k}}-\varepsilon_{k}=a_{k}, \quad \sum_{j=0}^{k-1} a_{j}=\frac{m_{k}}{n_{k}}, \quad \sum_{j=k+1}^{\infty} a_{j}+\varepsilon_{k}<f\left(n_{k}\right) .
$$

Here, $m_{k}$ and $n_{k}$ are not necessarily relatively prime, and $m_{k}$ is a function of $n_{k}$ and the rational number $\sum_{j=0}^{k-1} a_{j}$. Then

$$
a-\frac{b}{n_{k}}-\frac{m_{k}}{n_{k}}<\sum_{j=k+1}^{\infty} a_{j}+\varepsilon_{k}<f\left(n_{k}\right),
$$

where we define

$$
a=\sum_{j=0}^{\infty} a_{k}
$$

It is not difficult to see that the set of all $a$ which work is uncountable and dense in $(0,1)$.

4. Main examples and related results. It is natural to ask what are the behaviors of some power series in $U$, less artificial than those obtained in Theorems A and One concerning their exceptional points outside the unit circle and the corresponding limit sets. We first make the following observation concerning power series which are somewhat like the geometric series. Its proof is almost trivial and also follows trivially from a well-known theorem of Jentzsch [cf. 4].

Observation. Let $f$ be a function holomorphic in a neighborhood of the closed unit disc with an exception of a pole on the unit circle. Then the power series expansion of $f$ in $U$ has no exceptional point outside the unit circle.

If the function $f$ has more than one pole on the unit circle, then its power series expansion in $U$ may have exceptional points outside the unit circle as we shall see in the following theorems. In this direction, we should like to mention that, however, if $f$ has a finite number of algebraic singularities of fairly general type on the unit 
circle, then Shimizu [8] and Tsuji [9] have shown that the set of exceptional points of the power series expansion of $f$ in $U$ has linear measure zero. To obtain more specific informations, we examine the functions

$$
f_{\alpha}(z)=\frac{1}{1-z}-\frac{1}{1-z e^{i \alpha}}
$$

where $0<\alpha<2 \pi$, and let $P_{\alpha}$ denote the power series expansion of $f_{\alpha}$ in $U$. We obtain the following results.

THEOREM 4. Let $\alpha / 2 \pi$ be a rational number, say $\alpha / 2 \pi=p / q$ where $(p, q)=1$ and $0<p<q$. Let $n_{\alpha}=(q-p) / 2-1$ if $(q-p)$ is even and $n_{\alpha}=(q-p-1) / 2$ if $(q-p)$ is odd. Then the power series $P_{\alpha}$ has exactly $n_{\alpha}$ distinct exceptional points in $|z|>1$, where $n_{\alpha}$ may be zero. If $z^{*}$ is an exceptional point of $P_{\alpha}$, then $z^{*}=\mathrm{Re}^{-i \alpha / 2}$ for some $R>1$ and $L\left(z^{*}, P_{\alpha}\right)=\left\{f_{\alpha}\left(z^{*}\right)\right\}$. Furthermore, if $z_{0}=1+e^{-i \alpha}$ lies outside the unit circle, then it is one of these exceptional points.

THEOREM 5. Let $\alpha / 2 \pi$ be an irrational number. Then the power series $P_{\alpha}$ has an uncountable number of exceptional points in $|z|>1$. These points all lie on the ray $\mathrm{Re}^{-i \alpha / 2}$, where $1<R<\infty$, and form $a$ dense set of linear measure zero on this ray. Furthermore, if $z=\operatorname{Re}^{-i \alpha / 2}$ with $R>1$ and

$$
\lambda=\arg \left\{\left[1-\operatorname{Re}^{i \alpha / 2}\right] /\left[1-\operatorname{Re}^{-i \alpha / 2}\right]\right\},
$$

$0 \leqq \lambda<2 \pi$, then the limit set $L\left(z, P_{\alpha}\right)$ is contained in the straight line

$$
\left\{f(z)+\frac{i e^{-i \lambda / 2}}{1-z} x: x \text { real }\right\}
$$

We can be a little more precise about these limit sets, as in the following

THEOREM 6. For each irrational number $\alpha / 2 \pi$, the power series $P_{\alpha}$ has the property that the set of exceptional points $z=\mathrm{Re}^{-i \alpha / 2}$ with corresponding limit sets

$$
L\left(z, P_{\alpha}\right)=\left\{f_{\alpha}(z)+\frac{i e^{-i \lambda / 2}}{1-z} x: x \text { real }\right\}
$$

is uncountable, of measure zero and dense in the ray $\mathrm{Re}^{-i \alpha / 2}, R>1$, where $\lambda$ is as defined in the above theorem.

To prove these theorems, we consider the $(N+1)$ th partial sum of $P_{\alpha}$ : 


$$
\begin{aligned}
P_{N, \alpha}(z) & =\sum_{k=0}^{N}\left(1-e^{i k \alpha}\right) z^{k} \\
& =\frac{1-z^{N+1}}{1-z}-\frac{1-e^{i(N+1) \alpha} z^{N+1}}{1-z e^{i \alpha}} \\
& =f_{\alpha}(z)-\frac{z^{N+1}}{(1-z)\left(1-z e^{i \alpha}\right)}\left\{\left(1-z e^{i \alpha}\right)-e^{i(N+1) \alpha}(1-z)\right\} .
\end{aligned}
$$

Hence, for each $z$ with $|z|>1$, we see that $L\left(z, P_{\alpha}\right) \neq \varnothing$ if and only if there exists a sequence of positive integers $N_{j}$ such that

$$
\left(1-z e^{i \alpha}\right)-e^{i N j^{\alpha}}(1-z)=0\left(1 /|z|^{N_{j}}\right)
$$

as $N_{i} \rightarrow \infty$. Suppose that $|z|=R>1$ and (7) is satisfied. Then $e^{i N j^{\alpha}} \rightarrow e^{i \beta}=\left(1-z e^{i \alpha}\right) /(1-z)$ for some $\beta, 0 \leqq \beta \leqq 2 \pi$. Let $z=\operatorname{Re}^{i \theta}$. Then taking the arguments of both sides of the equality

$$
e^{i \beta}-1=\operatorname{Re}^{i(\theta+\alpha)}\left(e^{i(\beta-\alpha)}-1\right),
$$

we have

$$
(\beta+\pi) / 2=(\theta+\alpha)+(\beta-\alpha+\pi) / 2(\bmod 2 \pi),
$$

or $\theta=-\alpha / 2(\bmod 2 \pi)$. Hence, we conclude that if $z \neq \operatorname{Re}^{-i \alpha / 2}, R>1$, then $L\left(z, P_{\alpha}\right)=\varnothing$ for each $a, 0<a<2 \pi$. Thus, to find the exceptional points of $P_{\alpha}, 0<\alpha<2 \pi$, we only consider $z=\mathrm{Re}^{-i \alpha / 2}$ with $\mathrm{R}>1$. By (7), we see that $z=\mathrm{Re}^{-i \alpha / 2}, R>1$ is an exceptional point of $P_{\alpha}$ if and only if there is a sequence of positive integers $N_{j}$ such that

$$
e^{i j_{j} \alpha}-\frac{1-\mathrm{Re}^{i \alpha / 2}}{1-\mathrm{Re}^{-i \alpha / 2}}=0\left(1 / R^{N j}\right)
$$

as $N_{j} \rightarrow \infty$. Let

$$
e^{i \lambda}=\frac{1-\operatorname{Re}^{i \alpha / 2}}{1-\operatorname{Re}^{-i \alpha / 2}}, \quad 0 \leqq \lambda<2 \pi .
$$

Then it can be shown, by using some geometric argument, that $\lambda=\lambda(R)$ is a strictly decreasing continuously differentiable function on the interval $[1, \infty)$ with $\lambda(1)=\pi+\alpha / 2$ and $\lambda\left(\infty^{-}\right)=\alpha$. Also, it is clear that $\left|e^{i j^{\alpha} \alpha}-e^{i \lambda}\right|=0\left(1 / R^{N j}\right)$ if and only if

$$
\left|N_{j} \alpha / 2 \pi-\lambda / 2 \pi\right|(\bmod 1)=0\left(1 / R^{N_{j}}\right) \text {. }
$$

Let $a=\alpha / 2 \pi$ and $b=b(R)=\lambda(R) / 2 \pi, 0<a<1$. Then $z=\mathrm{Re}^{-i \alpha} / 2$ is an exceptional point of $P_{\alpha}$ if and only if

(I) $a<b<(a+1) / 2$, and

(II) there exist positive integers $N_{j}$ with

$$
\left(N_{j} a-b\right)(\bmod 1)=0\left(1 / R^{N_{j}}\right)
$$


as $N_{j} \rightarrow \infty$. Furthermore, the number of different values of $b=b(R)$ satisfying both (I) and (II) is exactly the same as the number of distinct exceptional points of $P_{\alpha}$ in $|z|>1$. The rest of the proofs of Theorems 4, 5, and 6 is now divided into two cases:

The rational case. Let $\alpha / 2 \pi=a=p / q$ be a rational number, where $p$ and $q$ are relatively prime with $0<p<q$. Then $n p / q(\bmod 1)$, $n=1,2, \cdots$, yields each $k / q(k=0, \cdots, q-1)$ infinitely often; while $k / q-b=0\left(1 / R^{n}\right)$ as $n \rightarrow \infty$ along a subsequence of positive integers if and only if $b=k / q(k=0, \cdots, q-1)$. Hence, the number of different values of $b$ satisfying (I) and (II) is the number of $k=$ $0, \cdots, q-1$ such that $p / q<k / q<(1+p / q) / 2$. This number is $n_{\alpha}$, which is $(q-p) / 2-1$ if $(q-p)$ is even and $(q-p-1) / 2$ if $(q-p)$ is odd. Also, since $k / q-b=0\left(1 / R^{n}\right)$ if and only if $k / q=b$, using (6), we see that if $z^{*}$ is an exceptional point of $P_{\alpha}$ then $L\left(z^{*}, P_{\alpha}\right)=$ $\left\{f_{\alpha}\left(z^{*}\right)\right\}$. Furthermore, if $z_{0}=1+e^{-i \alpha}$, then

$$
\begin{aligned}
P_{N, \alpha}\left(z_{0}\right) & =f_{\alpha}\left(z_{0}\right)-z_{0}^{N+1}\left\{\frac{1}{1-z_{0}}-\frac{e^{i(N+1) \alpha}}{1-z_{0} e^{i \alpha}}\right\} \\
& =f_{\alpha}\left(z_{0}\right)-z_{0}^{N+1} e^{i \alpha}\left\{e^{i(N-1) 2 \pi p / q}-1\right\}=f_{\alpha}\left(z_{0}\right)
\end{aligned}
$$

for $N=q-1,2 q-1,3 q-1, \cdots$. This completes the proof of Theorem 4 .

The irrational case. We now let $a=\alpha / 2 \pi$ be irrational, $0<a<1$. Since $\lambda(R)=2 \pi b=2 \pi b(R)$, it follows from (9) that $R(b)$ is a strictly decreasing continuously differentiable function on the interval $[a,(a+$ 1)/2] and has values greater than one there. We will use the $C^{1}$ property of $R(b)$ in the next section. Hence, we can apply Proposition 2 with $[r, s]=[a,(a+1) / 2]$, and conclude that the set of all exceptional points of $P_{\alpha}$ in $|z|>1$ is contained in the ray $\operatorname{Re}^{-i \alpha / 2}, R>1$, and is uncountable, of measure zero and dense on this ray.

Now, let $z=\mathrm{Re}^{-i \alpha / 2}, R>1$, be an exceptional point of $P_{\alpha}$. By (II) we see that there is a subsequence $N_{j}$ such that

$$
2 R^{N} \sin [(N \alpha-\lambda) / 2]
$$

converges to some real number $x$ as $N=N_{j} \rightarrow \infty$. But by (6),

$$
\begin{aligned}
P_{N-1, \alpha}(z) & =f_{\alpha}(z)+\frac{R^{N} e^{-i N \alpha / 2}}{1-z}\left(e^{i(N \alpha-\lambda)}-1\right) \\
& =f_{\alpha}(z)+\frac{i e^{-i N \alpha / 2} e^{i(N \alpha-\lambda) / 2}}{1-z} 2 R^{N} \sin [(N \alpha-\lambda) / 2] .
\end{aligned}
$$

Hence, we have 


$$
P_{N, \alpha}(z) \rightarrow f_{\alpha}(z)+\frac{i e^{-i \lambda / 2}}{1-z} x
$$

as $N=N_{j} \rightarrow \infty$, where $z=\operatorname{Re}^{-i \alpha / 2}$ and $\lambda=\lambda(R)$ is defined by $0 \leqq$ $\lambda<2 \pi$, and

$$
e^{i \curvearrowright}=\frac{1-\operatorname{Re}^{i \alpha / 2}}{1-\operatorname{Re}^{-i \alpha / 2}} .
$$

That is, we have proved that the limit set of $P_{\alpha}$ at $z=\operatorname{Re}^{-i \alpha / 2}$ is contained in the straight line

$$
\left\{f_{\alpha}(z)+\frac{i e^{-i \lambda / 2}}{1-z} x: x \text { real }\right\} \text {. }
$$

This completes the proof of Theorem 5 .

Let $L^{* *}(\lambda, \alpha)$ be the collection of all finite limit points of the convergent subsequences of the sequence

$$
k_{N}^{*}=2 R^{N} \sin [(N \alpha-\lambda) / 2] .
$$

Then as a corollary of Proposition 2, we see that the set of all $\lambda$, $\alpha<\lambda<\pi+\alpha / 2$, with $L^{* *}(\lambda, \alpha)=R$ is uncountable, of measure zero, and dense in $(\alpha, \pi+\alpha / 2)$. Hence, for each $\alpha$, the set of exceptional points $z=\operatorname{Re}^{-i \alpha / 2}, R>1$, such that

$$
L\left(z, P_{\alpha}\right)=\left\{f_{\alpha}(z)+\frac{i e^{-i \lambda / 2}}{1-z} x: x \in R\right\}
$$

is uncountable, of measure zero and dense on the ray $\mathrm{Re}^{-i \alpha / 2}, R>1$. This completes the proof of Theorem 6 .

Examples. By using Theorem 4, it follows that the power series $P_{\pi / 2}(z)=\sum_{k=0}^{\infty}\left(1-i^{k}\right) z^{k}$ has a unique exceptional point in $|z|>1$, namely at $1-i$, and the limit set of $P_{\pi / 2}$ at $1-i$ is $L\left(1-i, P_{\pi / 2}\right)=$ $\left\{f_{\pi / 2}(1-i)\right\}$. It also follows that $P_{2 \pi / 3}$ and $P_{\pi}$ have no exceptional points in $|z|>1$. It may be interesting to note that if $\alpha_{1}=$ $2 \pi(49 / 100), \alpha_{2}=2 \pi(499 / 1000), \cdots$, then $\alpha_{k} \rightarrow \pi$, and $P_{\alpha_{k}}$ has $2.5 \times 10^{k}$ exceptional points while the limit $P_{\pi}$ has none. On the other hand, by Theorem 5 , if $\alpha / 2 \pi$ is irrational and $\alpha_{k} / 2 \pi$ are rationals such that $\alpha_{k} \rightarrow \alpha$, then each $P_{\alpha_{k}}$ has a finite number of exceptional points, while the limit $P_{\alpha}$ has uncountably many exceptional points in $|z|>1$.

Finally, we remark that if $f$ is any function, holomorphic in a neighborhood of $|z| \leqq 1$ with an exception of two simple poles on $|z|=1$, then the power series of $f$ in $U$ has properties similar to those of $P$ in the above theorems, except that its exceptional points lie on a circle (which may be a straight line). If the two poles of $f$ are not simple but are of the same order, then the power series of $f$ 
may also have exceptional points in $|z|>1$, but if the orders of the two poles are different then it has no exceptional points in $|z|>1$.

5. Some improvement of Proposition 2 and Theorem 6. In the introduction we have stated that the sort of general result we like to obtain would say roughly that if a walk defined by a power series is active enough to have limit points, then the set of limit points ought to be "usually as large as possible". At first glance, Theorem 6 would support such a conjecture. Unfortunately, one may refine Proposition 2 to Proposition $2^{*}$ and conclude Theorem $6^{*}$ as below. For the setting, we refer the reader back to $\S 3$, and we assume further that $R(x)$ is continuously differentiable on $[r, s]$.

Proposition 2*. Every real number a, with an exception of a set of measure zero, has the property that if $S$ is any closed set on the real line then the set $\left\{b: L^{*}(b, a)=S\right\}$ is of cardinality $c$, of measure zero and dense on $[r, s]$.

As an immediate consequence, we have the following

THEOREM 6*. For almost all $\alpha$, the power series $P_{\alpha}$ has the property that if $S$ is any closed set on the real line then the set of exceptional points $z=\mathrm{Re}^{-i \alpha / 2}, R>1$, whose corresponding limit set is

$$
L\left(z, P_{\alpha}\right)=\left\{f_{\alpha}(z)+\frac{i e^{-i \lambda / 2}}{1-z} x: x \in S\right\},
$$

where $\lambda$ is as defined in Theorem 6, is uncountable and dense on the ray $\mathrm{Re}^{-i \alpha / 2}, R>1$.

We now devote the rest of the section to discuss our proof of Proposition $2^{*}$. If we were to proceed as in Proposition 2, letting $r_{1}, r_{2}, \cdots$ be a sequence of real numbers such that for each $N$, the set $\left\{r_{N}, r_{N+1}, \cdots\right\}$ is dense in $S$, we would find our $b$ 's such that $S \subseteq$ $L^{*}(b, a)$. In the proof of Proposition 2, we did not have to worry about picking up extra points since the limit set was full. The argument that for almost all $a$, the $b$ 's exist such that $S=L^{*}(b, a)$ is elementary but quite complicated. If we look at the proofs of Propositions 1 and 2, we see that the $b$ constructed was such that it is the intersection of the $I_{n}$, where $I_{1}, I_{2}, \ldots$ were defined inductively. To each $n>1$ in the induction, there corresponded an $n_{i-1}$ such that for any $x \in I_{i}$ (let us again always assume for convenience that $r_{i}>0$ ) such that for any $x \in I_{i}$

$$
\left(r_{i}-\frac{1}{i}\right) / R(x)^{n_{i-1}} \leqq\left\{n_{i-1} a\right\}-x \leqq\left(r_{i}+\frac{1}{i}\right) / R(x)^{n_{i-1}} \text {. }
$$


Now for $m$, such that $n_{i-1} \leqq m<n$, our potential $b$ is any point in $I_{n}$ and therefore our potential $k_{m}$ is in the set $K_{m}$ defined by

$$
K_{m}=\left\{t: \exists x \in I_{n} \text { such that } t / R(x)^{m}=\{m a\}-x\right\} .
$$

Thus, $S \subset L(b, a) \subset \bigcap_{N=1}^{\infty} \overline{\bigcup_{m=N}^{\infty} K_{m}}$. If we can insure that inf $\left\{|t|: t \in K_{m}\right\}$ goes to infinity as $m, m \neq n_{i}, i=1,2 \cdots$, tends to infinity, then

$$
\bigcap_{N=1}^{\infty} \overline{\bigcup_{m=N}^{\infty} K_{m}}=\bigcap_{N=1}^{\infty} \overline{\bigcup_{i=N}^{\infty} K_{n_{i}}}=S
$$

and we would have $L(b, a)=S$.

To give a punch to these ideas, we need a condition on $a$ to guarantee that the corresponding values $m a$ do not get too close to our potential $b$ 's too often. Thus, we now take a diversion to discuss what condition we need on $\alpha$. We state the following lemma without proof.

Lemma. Let a be a irrational number between 0 and 1 and let $k$ be a positive integer. Then

$$
\inf _{0 \leqq n<\infty} \inf _{0 \leqq p<q \leqq k}|\{(n+q) a\}-\{(n+p) a\}|=\inf _{0 \leqq p<q \leqq k} q\left|\frac{p}{q}-a\right|,
$$

where the infima are taken over integers.

This lemma makes it convenient to define a function

$$
\Psi_{a}(n)=\inf _{0 \leqq p<q \leqq n}|p-q a|
$$

where $a \in(0,1)$ is irrational. Then for each $a, \Psi_{a}(n)$ is a nonincreasing positive function of $n$ and it is not difficult to see that it approaches zero as $n$ tends to infinity. It is also not difficult to show that given a nonincreasing positive function $\bar{\Psi}$ defined on the positive integers, there is an irrational number $a$ such that $\Psi_{a}(n)<\bar{\Psi}(n)$ for infinitely many $n$. However, by a theorem in [1] (page 120) we know that if $\sum \bar{\Psi}(n)$ converges then the set

$$
\left\{a: \Psi_{a}(n) \leqq \bar{\Psi}(n) \text { for infinitely many } n\right\}
$$

is of measure zero. For $a \in(0,1)$ and a fixed $x$, we define $\phi_{a}(x)$ to be the smallest integer $n$ such that $\Psi_{a}(n) \leqq x$. By our previous remarks about $\Psi$, for any $\bar{\phi}(x)$, which increases as $x \downarrow 0$, there is an $a$ such that $\phi_{a}\left(x_{j}\right)>\bar{\phi}\left(x_{j}\right)$ for a sequence $x_{j} \downarrow 0$.

For instance, if $\bar{\Psi}(n)=1 / n^{2}$, then for almost all a, there exists $N_{a}$ such that $\Psi_{a}(n)>1 / n^{2}$ for $n \geqq N_{a}$; and for such an $a$, we have $\phi_{a}\left(1 / n^{2}\right) \geqq n$ for all sufficiently large $n$, and in particular, for all 
$n \geqq N_{a}$. For such $a$, Proposition $2 *$ holds. This ends the diversion and we continue the poof of Proposition $2^{*}$.

Let us proceed inductively on the $i$ corresponding to $I_{i}=\left[a_{i}, b_{i}\right]$, choosing the $n_{i}$ so large that the followings are satisfied. First, $n_{1}$ is so chosen that for $m \geqq n_{1}$ we have

$$
\dot{\phi}_{a}\left(1 / m^{2}\right) \geqq m \quad \text { and } \quad R(s)^{m}>10^{4} m^{5} .
$$

We again assume that $r_{i}>0$ and make sure that $0<r_{i} \leqq n_{i}$ for all $i$. Let

$$
D_{i}=\left[a_{i}-\left(10 n_{i-1}\right)^{-4}, b_{i}+\left(10 n_{i-1}\right)^{-4}\right]
$$

and $\lambda(J)$ stand for the length of the interval $J$. Then $\lambda\left(I_{i}\right) \leqq$ $2 / i R\left(b_{i}\right)^{n_{i-1}}$, and

$$
\lambda\left(D_{i}\right) \leqq \frac{2}{i R\left(b_{i}\right)^{n_{i-1}}}+\frac{2}{\left(10 n_{i-1}\right)^{4}} \leqq \frac{1}{n_{i-1}^{4}},
$$

so that $\dot{\phi}_{a}\left(\lambda\left(D_{i}\right)\right) \geqq n_{i-1}^{2}$. Thus, if $n_{i-1}<m<n_{i}$ and $\{m a\} \notin D_{i}$, then for any $t \in K_{m}$, there is an $x \in I_{i}$ such that

$$
|t|=|\{m a\}-x| R(x)^{m} \geqq R(s)^{m} /\left(10 n_{i-1}\right)^{4} \geqq m .
$$

Therefore, $|t|$ goes to infinity with $m$ and we need only worry about $m$ when $\{m a\} \in D_{i}$. Suppose $m_{1}=n_{i-1}+k$ is the smallest integer greater than $n_{i-1}$ that lies in $D_{i}$. Then if $\left\{m_{1} a\right\},\left\{m_{1} a\right\}-\left(r_{i}+1 / i\right) / R\left(b_{i}\right)^{m_{1}}$, $\left\{m_{1} a\right\}-\left(r_{i}-1 / i\right) / R\left(b_{i}\right)^{m_{1}},\left\{m_{1} a\right\}-\left(r_{i}+1 / i\right) / R\left(a_{i}\right)^{m_{1}}$, and $\left\{m_{1} a\right\}-\left(r_{i}-\right.$ $1 / i) / R\left(a_{i}\right)^{m_{1}}$ all lie in $I_{i}$, we can proceed as in the proofs of Propositions 1 and 2 . If these conditions are not satisfied, then by cutting off a section of the interval $I_{i}$ of length $5 i / R\left(b_{i}\right)^{m_{1}}$ from one end or the other, we achieve a new interval $I_{i}^{\prime}=\left[a_{i}^{\prime}, b_{i}^{\prime}\right] \subset I_{i} \subset D_{i}$, such that for $x \in I_{i}^{\prime}$ and $t \in K_{m_{i}}$, then $|t| \geqq i$.

If $\left\{m_{1} a\right\} \in\left[a_{i}+5 i / 2 R(s)^{m_{1}}, b_{i}-5 i / 2 R(s)^{m_{1}}\right]$, then the five points mentioned above lie in $I_{i}$ and the induction could be completed as in Propositions 1 and 2. Otherwise, we get $I_{i}^{\prime}$ by cutting $I_{i}$ from the end of $a_{i}$ or $b_{i}$, whichever is closer to $\left\{m_{1} a\right\}$. We now proceed to the next integer $m_{2}$ such that $\left\{m_{2} a\right\} \in D_{i}$ and test to see if the induction can proceed as described by Propositions 1 and 2 with $I_{i}^{\prime}$ in place of $I_{i}$, or we perform another cutting of $I_{i}^{\prime}$ to form $I_{i}^{\prime \prime}$ by cutting off a piece of length $5 i / R\left(b_{i}\right)^{m_{2}}$. We continue with this second induction until we find that we are in a situation in which we can define $I_{i+1}$ as in Propositions 1 and 2. To see that we must reach such a stage, let us add up the pieces cut off if the induction on the $j$ of $m_{j}$ continues with $i$ held fixed. The total length of the pieces cut off is less than 


$$
\sum_{j=1}^{\infty} \frac{5 i}{R\left(b_{i}\right)^{m}} \leqq \frac{5 i}{R\left(b_{i}\right)^{n_{i-1}^{2}}} \sum_{k=0}^{\infty} \frac{1}{R\left(b_{i}\right)^{k\left(n_{i}-1\right)^{2}}} \leqq \frac{10 i}{R\left(b_{i}\right)^{n_{i-1}^{2}}} .
$$

Therefore, since the $\{n a\}$ are dense in $[0,1]$, the second induction must end provided only we can show that

$$
\lambda\left(I_{i}\right)>10 i / R\left(b_{i}\right)^{n^{2}}{ }_{i-1} .
$$

If we go back to Proposition 1, we see that we can take $\lambda\left(I_{i}\right)$ to be

$$
b_{i}-a_{i}=\left[\left\{n_{i-1} a\right\}-\frac{r_{i}-1 / i}{R\left(b_{i}\right)^{n_{i-1}}}\right]-\left[\left\{n_{i-1} a\right\}-\frac{r_{i}+1 / i}{R\left(a_{i}\right)^{n_{i-1}}}\right] .
$$

For instance, if $R\left(a_{i}\right)=R\left(b_{i}\right)=R>0$, then $b_{i}-a_{i}=2 / i R^{n_{i-1}}$ which is clearly greater than $10 i / R\left(b_{i}\right)^{n_{i-1}^{2}}$ for large values of $i$. However, if $R\left(a_{i}\right) \neq R\left(b_{i}\right)$, we need a more delicate argument. To do this, let us define the following three functions

$$
\begin{aligned}
& f_{1}(x)=\left\{n_{i-1} a\right\}-\left(r_{i}-\frac{1}{i}\right) / R(x)^{n_{i-1}}, \\
& f_{2}(x)=\left\{n_{i-1} a\right\}-r_{i} / R(x)^{n_{i-1}}, \\
& f_{3}(x)=\left\{n_{i-1} a\right\}-\left(r_{i}+\frac{1}{i}\right) / R(x)^{n_{i-1}},
\end{aligned}
$$

and consider their graphs on $I_{i}^{(k)}=\left[a_{i}^{(k)}, b_{i}^{(k)}\right]$ which we shall call $I_{i}=$ $\left[\alpha_{i}, b_{i}\right]$ for convenience. Then for each $i$, we have $f_{1}\left(b_{i}\right) \leqq b_{i}$ and $f_{3}\left(a_{i}\right) \geqq a_{i}$, so that there is an $a^{\prime} \in I_{i}$ such that $f_{2}\left(a^{\prime}\right)=a^{\prime}$.

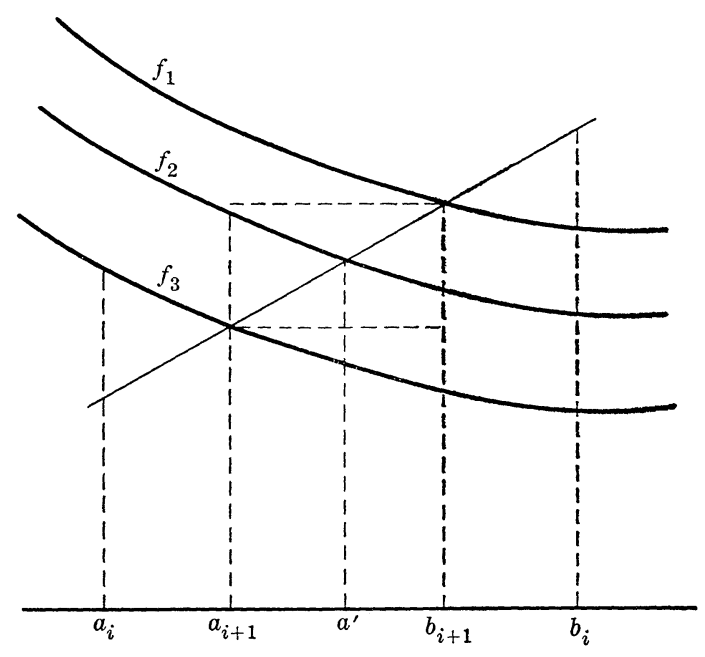

Let $I_{i+1}$ be determined by the largest rectangle lying between $f_{1}$ and $f_{3}$ with the line $f(x)=x$ as diagonal (cf. the above figure). Then $\lambda\left(I_{i+1}\right)$ is not too far from $2 R\left(a^{\prime}\right)^{-n} /(i+1)$. For instance, if we assume 
that $R(x)$ is continuously differentiable and $n_{i}$ is large enough, elementary estimates give $\lambda\left(L_{i+1}\right)>1 /\left[2(i+1) R\left(a^{\prime}\right)^{n}\right]$ (since the derivatives are very close to zero). It seems that the hypothesis of continuous differentiability is unnecessary but we do not press the matter to remove it. However, we do not know whether we can do this for all $a$.

6. Final remarks. In conclusion, we propose some problems and try to justify a statement in our introduction.

If $P$ is a power series in $U$, the set of all exceptional points of $P$ in $|z|>1$ will be called the exceptional set of $P$. One question is: What are the possible exceptional sets? It is easy to see that a necessary condition for a set $S$ to be exceptional set of a power series $P$ in $U$ is that $S$ is both a $G_{\delta \sigma o}$ and $F_{\sigma \dot{\sigma} \sigma}$ set. This follows since $S$ must be the complement of a set of the types,

$$
\bigcap_{M} \bigcup_{k} \bigcap_{n \geqq k}\left\{z:|z|>1 \text {, and }\left|P_{n}(z)\right|>M\right\}
$$

and

$$
\bigcap_{M} \bigcup_{k} \bigcap_{n \geqq k}\left\{z:|z|>1 \text { and }\left|P_{n}(z)\right| \geqq M\right\} \text {. }
$$

Examples in this paper show that $S$ may be very complicated.

As mentioned in the first section, the spirit of this paper is that of looking at examples of random walks in the complex plane with large steps. If the steps go to infinity slowly enough, it is not hard to see that under reasonable general conditions, one gets that the set of nonexceptional points is of measure zero; in fact, almost all points would have the property that the walk enters every neighborhood infinitely often. Also, one can put conditions on the growth steps to guarantee that no finite neighborhood is entered infinitely often. On the other hand, there are conditions under which the growth of the steps is such that one can expect the set of exceptional points to be of measure zero. Suppose we restrict ourselves to this latter case. Are there any probabilistic statements one can make relative to the set of exceptional points? For instance, let us suppose that we have a power series $P(z)$ in $U$ and let $f(z)$ be its analytic continuation to the largest possible domain in the plane. Let $B(P)$, the set of exceptional points of $P(z)$, be of measure zero. What is the relative probability (relative to $B(P)$ ) of those points whose limit sets consist only of values gotten by analytic continuation, and what is the relative probability that the limit set is a particular closed set?

Most of this paper deals with the "simplest" examples which may give a hint to the answers to these types of questions, namely the case where $P(z)=P_{\alpha}(z)$ and the closely related results on diophantine 
approximations. For instance, it may be conjectured that in some probabilistic sense, the set $\{z: L(z, P)=\{f(z)\}\}$ is "larger" than the set $\{z: L(z, P)=\{p\}\}$ where $p$ is any point different from $f(z)$. This conjecture seems to be supported by Theorem 4 and the classical view point of overconvergence. Another possible conjecture is roughly that $L(z, P)$ is probably as large as it can be. This is what we intuitively take as the idea of fullness.

We close with a result, namely Theorem 7, to try to justify our feeling to the above statements. We first state a result obtained in [7].

Lemma. There exists a function $\rho(A, B)$ defined for any subset $A$ of $[0,1]$ and any nonempty set $B$ with the following properties:

(a) $\rho(A, A)=1, \quad 0 \leqq \rho(A, B) \leqq 1$,

(b) $\rho(A, B)=\rho(A \cap B, B)$,

(c) $\rho(A \cup B, C)=\rho(A, C)+\rho(B, C)$,

(d) if $A \subset B \subset C, \rho(A, B) \rho(B, C)=\rho(A, C)$,

(e ) if $A$ and $B$ are Lebesgue measureable and $\lambda$ is the Lebesgue measure then $\rho(A, B) \lambda(B)=\lambda(A \cap B)$ and

(f) $\rho(A+\alpha, B+\beta)=\rho(A, B)$, if $A \subset B$ and $A+\alpha \subset B+\beta$ (addition is taken $\bmod 1)$.

Let $R>1$ and $a$ be any irrational on $(0,1)$. For a closed set $E$, let $B(a, R, E)=\left\{b: L^{*}(b, a)=E\right\}$, and let $\theta_{R}(a)=\bigcup_{E} B(a, R, E)$ (cf. definition in $\S 3$ ).

THeOREM 7. Let $E$ be a closed set with $R E \neq E$ and let $\rho$ be any conditional probability function as described in the above lemma. Then

$$
\rho\left(B(\alpha, R, E), \quad \theta_{R}(\alpha)\right)=0 \text {. }
$$

To prove this, we let $b \in B(a, R, E)$. Then $L^{*}(b, a)=E$. Write $k_{n}=(\{n a\}-b) R^{n}$. Then $E$ is the set of limit points of $k_{n}$. Consider $L^{*}(a+b, a)$ and define $\widetilde{k}_{n}=(\{n a\}-(a+b)) R^{n}$. For a subsequence of $n, \widetilde{k}_{n}=(\{(n-1) a\}-b) R^{n}$, so that $\widetilde{k}_{n}=R k_{n-1}$. Thus, $L^{*}(b+a, a)=$ $R L^{*}(b, a)$ and

$$
a+B(\alpha, R, E) \subseteq B(\alpha, R, R E), \quad 2 a+B(\alpha, R, E) \subseteq B\left(a, R, R^{2} E\right), \cdots,
$$

$m a+B(a, R, E) \subseteq B\left(a, R, R^{m} E\right), \cdots$. By the translation invariance of $\rho$, we have $\rho\left(m a+B(a, R, E), \theta_{R}(a)\right)=\rho\left(B(a, R, E), \theta_{R}(a)\right)$ for all $m$. Since $B\left(a, R, R^{m} E\right), m=0,1, \cdots$, are pairwise disjoint, we have, by finite additivity in the first coordinate of $\rho$,

$$
\begin{aligned}
k \rho\left(B(a, B, E), \theta_{R}(a)\right) & =\sum_{m=1}^{k} \rho\left(m a+B(a, R, E), \theta_{R}(a)\right) \\
& =\rho\left(\bigcup_{m=1}^{k}(m a+B(a, R, E)), \theta_{R}(a)\right) \leqq 1 .
\end{aligned}
$$


This holds for all $k$, and hence, $\rho\left(B(a, R, E), \theta_{R}(a)\right)=0$.

We want to point out that the case $E=\{0\}$ is closely related to the classical interpretation of overconvergence and the case where $E=R E$ is closely related to our idea of fullness.

\section{REFERENCES}

1. J.W.S. Cassels, An Introduction to Diophantine Approximation, Cambridge University Press, 1957.

2. C. K. Chui and M. N. Parnes, Approximation by overconvergence of a power series, J. Math. Analysis and Applications, 36 (1971), 692-696.

3. K. L. Chung and W. H. J. Fuchs, On the distribution of values of sums of random variables, Memoirs, of the American Mathematical Society, No. 6, 1951.

4. P. Dienes, The Taylor Series. Oxford University Press, 1931.

5. E. Hille, Analytic Function Theory, Vol. 2, Ginn and Co., 1962.

6. R. Parikh and M. N. Parnes, Conditional probability can be defined for all pairs of sets of reals, Advances in Mathematics, 9 (1972), 313-315.

7. - Conditional Probability and Uniform Sets I, Springer Lecture Note Series, Proc. Conference on Nonstandard Analysis, Victoria, B. C., 1972, P. Loeb, Editor. To appear.

8. T. Shimizu, On some power series and their sections II, Japan J. Math., 5 (1928), $153-162$.

9. M. Tsuji, On a power series which has only algebraic singularities on its convergence circle IV, Japan J. Math., 5 (1928), 163-184.

Received February 16, 1973.

TEXAS A \& M UNIVERSity

AND

State University of New York at Buffalo 



\section{PACIFIC JOURNAL OF MATHEMATICS}

\section{EDITORS}

RICHARD ARENS (Managing Editor)

University of California

Los Angeles, California 90024

\section{R. A. Beaumont \\ University of Washington \\ Seattle, Washington 98105}

\section{J. DugundjI*}

Department of Mathematics University of Southern California Los Angeles, California 90007

D. Gilbarg and J. Milgram

Stanford University

Stanford, California 94305

\section{ASSOCIATE EDITORS}
E. F. BeCKenBaCH
B. H. NeumanN
F. WOLF
K. YoSHIDA

\section{SUPPORTING INSTITUTIONS}

\author{
UNIVERSITY OF BRITISH COLUMBIA \\ CALIFORNIA INSTITUTE OF TECHNOLOGY \\ UNIVERSITY OF CALIFORNIA \\ MONTANA STATE UNIVERSITY \\ UNIVERSITY OF NEVADA \\ NEW MEXICO STATE UNIVERSITY \\ OREGON STATE UNIVERSITY \\ UNIVERSITY OF OREGON \\ OSAKA UNIVERSITY
}

\author{
UNIVERSITY OF SOUTHERN CALIFORNIA \\ STANFORD UNIVERSITY \\ UNIVERSITY OF TOKYO \\ UNIVERSITY OF UTAH \\ WASHINGTON STATE UNIVERSITY \\ UNIVERSITY OF WASHINGTON

$* * *$
$*$
AMERICAN MATHEMATICAL SOCIETY
NAVAL WEAPONS CENTER

The Supporting Institutions listed above contribute to the cost of publication of this Journal, but they are not owners or publishers and have no responsibility for its content or policies.

Mathematical papers intended for publication in the Pacific Journal of Mathematics should be in typed form or offset-reproduced, (not dittoed), double spaced with large margins. Underline Greek letters in red, German in green, and script in blue. The first paragraph or two must be capable of being used separately as a synopsis of the entire paper. Items of the bibliography should not be cited there unless absolutely necessary, in which case they must be identified by author and Journal, rather than by item number. Manuscripts, in duplicate if possible, may be sent to any one of the four editors. Please classify according to the scheme of Math. Rev. Index to Vol. 39. All other communications to the editors should be addressed to the managing editor, or Elaine Barth, University of California, Los Angeles, California, 90024.

100 reprints are provided free for each article, only if page charges have been substantially paid. Additional copies may be obtained at cost in multiples of 50 .

The Pacific Journal of Mathematics is issued monthly as of January 1966. Regular subscription rate: $\$ 60.00$ a year (6 Vols., 12 issues). Special rate: $\$ 30.00$ a year to individual members of supporting institutions.

Subscriptions, orders for back numbers, and changes of address should be sent to Pacific Journal of Mathematics, 103 Highland Boulevard, Berkeley, California, 94708.

PUBLISHED BY PACIFIC JOURNAL OF MATHEMATICS, A NON-PROFIT CORPORATION

Printed at Kokusai Bunken Insatsusha (International Academic Printing Co., Ltd.), 270, 3-chome Totsuka-cho, Shinjuku-ku, Tokyo 160, Japan

* C. R. DePrima California Institute of Technology, Pasadena, CA 91109, will replace J. Dugundji until August 1974. 


\section{Pacific Journal of Mathematics}

Vol. 50, No. $2 \quad$ October, 1974

Mustafa Agah Akcoglu, John Philip Huneke and Hermann Rost, A counter example to the Blum Hanson theorem in general spaces .............

Huzihiro Araki, Some properties of modular conjugation operator of von

Neumann algebras and a non-commutative Radon-Nikodym theorem

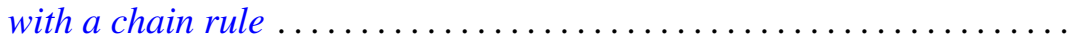

E. F. Beckenbach, Fook H. Eng and Richard Edward Tafel, Global properties of rational and logarithmico-rational minimal surfaces .....

David W. Boyd, A new class of infinite sphere packings ............. 383

K. G. Choo, Whitehead Groups of twisted free associative algebras ........

Charles Kam-Tai Chui and Milton N. Parnes, Limit sets of power series

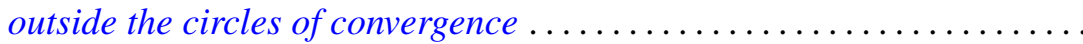

Allan Clark and John Harwood Ewing, The realization of polynomial

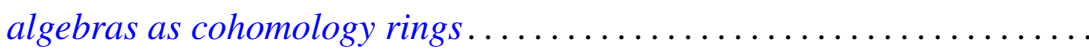

Dennis Garbanati, Classes of circulants over the p-adic and rational

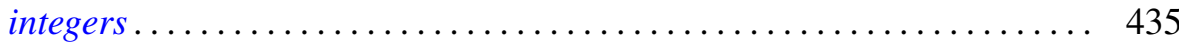

Arjun K. Gupta, On a "square" functional equation ................... 449

David James Hallenbeck and Thomas Harold MacGregor, Subordination and extreme-point theory ............................. 455

Douglas Harris, The local compactness of $v X \ldots \ldots . . . . . . . . . . . .4469$

William Emery Haver, Monotone mappings of a two-disk onto itself which fix the disk's boundary can be canonically approximated by homeomorphisms .................................. 477

Norman Peter Herzberg, On a problem of Hurwitz .................. 485

Chin-Shui Hsu, A class of Abelian groups closed under direct limits and subgroups formation ............................... 495

Bjarni Jónsson and Thomas Paul Whaley, Congruence relations and multiplicity types of algebras.....................

Lowell Duane Loveland, Vertically countable spheres and their wild sets.

Nimrod Megiddo, Kernels of compound games with simple components ....

Russell L. Merris, An identity for matrix functions ........ . .

E. O. Milton, Fourier transforms of odd and even tempered distributions ...

Dix Hayes Pettey, One-one-mappings onto locally connected generalized continua

Mark Bernard Ramras, Orders with finite global dimension

Doron Ravdin, Various types of local homogeneity. .

George Michael Reed, On metrizability of complete Moore spaces ...

Charles Small, Normal bases for quadratic extensions ..

Philip C. Tonne, Polynomials and Hausdorff matrices.... . . 